University of Nebraska - Lincoln

DigitalCommons@University of Nebraska - Lincoln

Faculty Publications from Nebraska Center for Materials and Nanoscience, Nebraska Center Materials and Nanoscience

$12-29-2006$

\title{
Wave propagation in nanofibers
}

Xiangfa Wu

University of Nebraska - Lincoln, xwu3@unl.edu

Yuris A. Dzenis

University of Nebraska-Lincoln, ydzenis@unl.edu

Follow this and additional works at: https://digitalcommons.unl.edu/cmrafacpub

Part of the Nanoscience and Nanotechnology Commons

Wu, Xiangfa and Dzenis, Yuris A., "Wave propagation in nanofibers" (2006). Faculty Publications from Nebraska Center for Materials and Nanoscience. 31.

https://digitalcommons.unl.edu/cmrafacpub/31

This Article is brought to you for free and open access by the Materials and Nanoscience, Nebraska Center for (NCMN) at DigitalCommons@University of Nebraska - Lincoln. It has been accepted for inclusion in Faculty Publications from Nebraska Center for Materials and Nanoscience by an authorized administrator of DigitalCommons@University of Nebraska - Lincoln. 


\title{
Wave propagation in nanofibers
}

\author{
Xiang-Fa Wu ${ }^{\text {a) }}$ and Yuris A. Dzenis ${ }^{\text {b) }}$ \\ Department of Engineering Mechanics, National Nanofiber Facility, \\ University of Nebraska-Lincoln, Lincoln, Nebraska 68588-0526
}

(Received 22 August 2006; accepted 21 October 2006; published online 29 December 2006)

\begin{abstract}
Surface effect on the longitudinal and flexural wave propagation in straight nanofibers/nanowires is studied within the framework of continuum mechanics. Modified Euler-Bernoulli and Rayleigh-Love rod equations and modified Timoshenko beam equation were developed to take into account the surface effect. It was found that surface tension/stress plays appreciable influence on the wave propagation in nanofibers/nanowires at very small diameters. Wave dispersion relations were presented in closed form, and numerical calculations were performed to show the variation of the phase velocity with the wave number at varying surface property and fiber diameter. The given results can be used as the theoretical basis of dynamic characterization of nanofibers/nanowires and nanodevice design and analysis. (C) 2006 American Institute of Physics. [DOI: 10.1063/1.2403976]
\end{abstract}

\section{INTRODUCTION}

Surface has appreciable effect on material properties of metallic and organic wires/fibers and films in nanoscale as observed in experiments and predicted in theoretical studies. ${ }^{1-7}$ For crystalline nanowires and ultrathin films surface stresses may either increase or decrease the corresponding surface moduli depending on the specific material types and crystalline microstructures, ${ }^{4}$ and surface tension may lead to rupture of ultrathin polymer films. ${ }^{8,9}$ As a result, these unique surface properties may further influence other physical properties such as the surface wave speed, thermal and electrical conductivities, etc. Therefore, the study of dynamic properties of materials and structures in nanoscale will play an important role in nanotechnologies, e.g., dynamic characterization of nanofibers/nanowires, dynamic design, analysis, and calibration of nanodevices and nanostructures, among others. Dynamic properties of materials in nanoscale have attracted significant attention in recent years. For onedimensional (1D) carbon nanotubes, recent increasing investigations have shown the size effect on their wave propagation within the framework of continuum elastic shell theory ${ }^{10-14}$ molecular dynamics, ${ }^{15,16}$ molecular structural mechanics, ${ }^{17}$ etc. These investigations have indicated numerous interesting phenomena of wave propagation in carbon nanotubes that are different from those found in the classic wave theories for elastic rods and beams. For example, by means of modified elastic shell theory, Yoon et al. ${ }^{10}$ indicated the transition of coaxial and noncoaxial modes of transverse sound wave propagating in individual multiwall carbon nanotubes at specific frequency thresholds within the terahertz range. Using molecular structural mechanics based on the classic finite element method formulation, $\mathrm{Li}$ and $\mathrm{Chou}^{17}$ found that the longitudinal wave speed in single-wall carbon nanotubes is roughly doubled by comparison with that of the torsional waves, and the transverse waves are dispersive and dependent on the nanotube geometries. These wave motion

\footnotetext{
a) Electronic mail: xfwu@unlserve.unl.edu

${ }^{b)}$ Electronic mail: ydzenis@unl.edu
}

properties may be used for the purpose of carbon nanotube alignment, ${ }^{18}$ nanotube-based nanodevice design and calibration, nonstructural analysis, etc.

Continuous nanofibers produced by electrospinning technique are emerging as a new type of 1D nano- and submicron materials. ${ }^{1920}$ Their continuity, super high surface area to volume ratio, low-cost, and high flexibility in fabrication have evoked increasing interest in producing nanofibers from various precursor materials for broad applications in filtration, chemical catalyst carriers, biomedical templates, nanocomposites, etc. ${ }^{21,22}$ Continuous nanofibers with very small diameter $(<5 \mathrm{~nm})$, such as recently electrospun nylon4,6 nanofibers, ${ }^{23}$ have been fabricated successfully in laboratory, and mechanical properties of electrospun nanofibers have attracted significant attention on their characterization and modeling. ${ }^{24-26}$ Theoretically, early $a b$ initio all-electron calculations for carbon and oxygen chemisorbed on nickel clusters indicated that the resulting surface stresses modified the surface energy and surface wave propagation. ${ }^{27}$ However, no work has been reported yet regarding the wave propagation in nanofibers/nanowires. Without a doubt, dynamic properties of nanofibers/nanowires may have some unique characteristics closely related to their nanoscale geometries. Thus, in the present paper we first initiate the study to consider the surface tension/stress effect on the longitudinal and flexural wave propagation in nanofibers/nanowires within the framework of continuum mechanics. Modified Euler-Bernoulli and Rayleigh-Love rod equations and modified Timoshenko beam equation are developed to take into account the effect of the surface tension/stress in the first order. It is found that surface tension/stress plays appreciable role in the wave propagation in the nanofibers/nanowires with very small diameters. The corresponding wave dispersion relations are given in closed form. Numerical calculations are performed to show the variation of the phase velocity with the wave number at varying surface property and fiber diameter. 


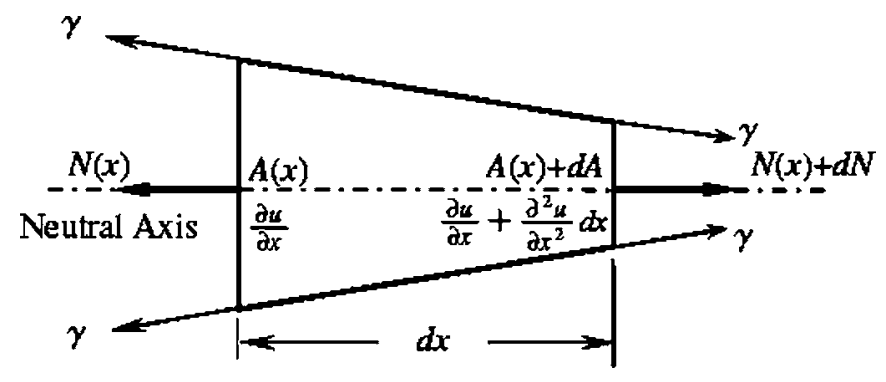

FIG. 1. Representative nanofiber element in longitudinal wave propagation.

\section{PROBLEM STATEMENT AND SOLUTION}

\section{A. Longitudinal wave propagation}

Consider the longitudinal wave propagating in a straight elastic nanofiber/nanowire with constant cross section. The free body diagram of a representative element of the nanofiber/nanowire is illustrated in Fig. 1, where the $x$ axis is selected along the nanofiber/nanowire axis. In this case, the motion of the nanofiber/nanowire is axisymmetric. Though prestress is commonly employed in measurement and nanodevices, its effect on the longitudinal wave propagation is in the sense of a higher order term compared to that of the surface tension/stress and therefore is ignored. The symbol $\gamma$ used in Fig. 1 indicates either the surface tension for polymer nanofibers or the surface stress for metallic nanowires. The dynamic equilibrium of the nanofiber/nanowire element in the first order leads to

$$
\frac{\partial^{2} u}{\partial t^{2}}=\left(c^{2}-\frac{2 \nu \gamma}{\rho R_{0}}\right) \frac{\partial^{2} u}{\partial x^{2}},
$$

where $u(x, t)$ is the axial displacement, $c=\sqrt{E / \rho}$ is the longitudinal wave speed corresponding to the bulk material with $\rho$ mass density and $E$ Young's modulus, $\nu$ is Poisson's ratio, and $R_{0}$ is the radius of the nanofiber/nanowire at rest. Equation (1) is the modified Euler-Bernoulli rod equation. Furthermore, since dynamic nanodevices potentially with nanofibers/nanowires are expected to work in the highfrequency range, the transverse inertia may have appreciable effect on their dynamic responses. Thus, by taking into account the transverse inertia effect, the resulting modified Rayleigh-Love rod equation reads

$$
\frac{\partial^{2} u}{\partial t^{2}}=\left(c^{2}-\frac{2 \nu \gamma}{\rho R_{0}}\right) \frac{\partial^{2} u}{\partial x^{2}}+\frac{\nu^{2} R_{0}^{2}}{2} \frac{\partial^{4} u}{\partial t^{2} \partial x^{2}} .
$$

The wave equations (1) and (2) indicate that the longitudinal wave propagation is closely related to the diameter of the nanofiber/nanowire. At small diameters, the surface tension has remarkable influence on the wave motion, while at great diameters the transverse inertia has appreciable effect on the wave motion at high frequencies. To derive the wave dispersion relation, consider the harmonic wave

$$
u(x, t)=A \exp [i k(x-V t)]
$$

where $A$ is the wave amplitude, $k$ the wave number, $V$ the phase velocity, and $i=\sqrt{-1}$. Substitution of (3) into (1) leads to the phase velocity

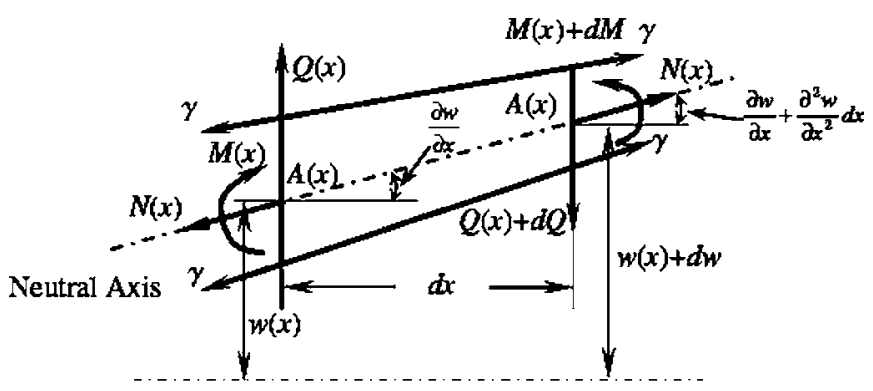

Equilibrium Position

FIG. 2. Representative nanofiber element in flexural wave propagation.

$$
V=\sqrt{c^{2}-2 \nu \gamma /\left(\rho R_{0}\right)} .
$$

The wave propagation in this case is nondispersive, and the wave velocity decreases with the decrease of the nanofiber/ wire radius, especially at very small radius, i.e., it has the size effect. With consideration of the transverse inertia effect, substitution of (3) into (2) yields the wave dispersion relation as

$$
\left[c^{2}-2 \nu \gamma /\left(\rho R_{0}\right)\right] k^{2}=\omega^{2}\left(1+\nu^{2} R_{0}^{2} k^{2} / 2\right),
$$

where $\omega=\mathrm{kV}$ is the circular frequency. In this case, the wave propagation is dispersive. Relation (5) can be rewritten such that

$$
\frac{\omega}{k}=\frac{\sqrt{c^{2}-2 \nu \gamma /\left(\rho R_{0}\right)}}{\sqrt{1+\nu^{2} R_{0}^{2} k^{2} / 2}} .
$$

For small wave number $k$, relation (6) can be expanded in terms of a Taylor series with respect to $k^{2}$. The first two terms of this series form the modified Pochhammer frequency equation as

$$
\frac{\omega}{k}=\left[c^{2}-2 \nu \gamma /\left(\rho R_{0}\right)\right]^{1 / 2}\left(1-\nu^{2} R_{0}^{2} k^{2} / 4\right) .
$$

\section{B. Flexural wave propagation}

Now let us further consider the flexural wave propagation in prestressed nanofibers/nanowires. In this case, the free-body diagram of a representative element is illustrated in Fig. 2. The transient deflection $w(x)$ is measured from the neutral axis of the nanofiber/nanowire at rest to that at the transient position. Hereafter, we derive the wave equation based on the Timoshenko beam that takes into account the shear effect. Besides the surface tension/stress, the nanofiber/ nanowire is also assumed being prestressed at prestrain $\varepsilon_{0}$. To set up the flexural wave equation of the microbeam, we need the bending formula,

$$
M=E I \kappa,
$$

where $M=\int_{A} \sigma_{x} y d A$ is the bending moment, $I=\int_{A} y^{2} d A$ $=\pi R_{0}^{4} / 4$ is the moment of inertia of the nanofiber/nanowire cross section with respect to the neural axis, and $\kappa=1 / \rho$ $=d \varphi / d s$ is the curvature of the neutral axis after bending. Here $\varphi$ is the angle due to the pure bending moment. In the case of small deflection, the beam curvature can be approximated as 


$$
\kappa=\frac{d \varphi}{d s} \approx \frac{d \varphi}{d x} .
$$

Furthermore, the assumption of Timoshenko beam says that the total slope $\theta=\partial w / \partial x$ of the beam is the sum of the slope by the pure bending and the slope by the shear force,

$$
\frac{\partial w}{\partial x}=\frac{\partial w_{b}}{\partial x}-\frac{\partial w_{s}}{\partial x}
$$

i.e.,

$$
\theta=\varphi-\alpha,
$$

where $\varphi=\partial w_{b} / \partial x$ and $\alpha=\partial w_{s} / \partial x$, and the subscripts $b$ and $s$ indicate the beam deflections due to pure bending and shear deformation, respectively. Thus, the force-deformation relations can be expressed as

$$
\begin{aligned}
& M=E I \frac{\partial \varphi}{\partial x}, \\
& Q=\beta A G\left(\varphi-\frac{\partial w}{\partial x}\right),
\end{aligned}
$$

where $\beta$ is the shape coefficient of the Timoshenko beam with circular cross section, ${ }^{28}$

$$
\beta=\frac{6+12 \nu+6 \nu^{2}}{7+12 \nu+4 \nu^{2}},
$$

$A=\pi R_{0}^{2}$ is the cross-sectional area, and $G$ is the shear modulus. Consequently, with consideration of prestrain $\varepsilon_{0}$ and surface tension/stress $\gamma$, relations (12) and (13) lead to the dynamic equations for the nanofiber/nanowire based on the element of length $d x$ (see Fig. 2) subjected to the bending moment $M$ and shear force $Q$,

$$
\begin{aligned}
& \rho A \frac{\partial^{2} w}{\partial t^{2}}+\frac{\partial Q}{\partial x}-\left(E A \varepsilon_{0}+2 \pi R_{0} \gamma\right) \frac{\partial^{2} w}{\partial x^{2}}=0, \\
& \rho I \frac{\partial^{2} \varphi}{\partial t^{2}}+Q-\left(E A \varepsilon_{0}+2 \pi R_{0} \gamma\right) \frac{\partial w}{\partial x}-\frac{\partial M}{\partial x}=0 .
\end{aligned}
$$

Substitution of (12) and (13) into (15) and (16) leads to a set of dynamic equations governing the wave motion,

$\rho \frac{\partial^{2} w}{\partial t^{2}}+\beta G\left(\frac{\partial \varphi}{\partial x}-\frac{\partial^{2} w}{\partial x^{2}}\right)-\left(E \varepsilon_{0}+\frac{2 \gamma}{R_{0}}\right) \frac{\partial^{2} w}{\partial x^{2}}=0$,

$\rho I \frac{\partial^{2} \varphi}{\partial t^{2}}+\beta A G\left(\varphi-\frac{\partial w}{\partial x}\right)-\left(E A \varepsilon_{0}+2 \pi R_{0} \gamma\right) \frac{\partial w}{\partial x}-E I \frac{\partial^{2} \varphi}{\partial x^{2}}=0$.

By assuming the wave motion of the nanofiber/nanowire as

$$
w(x, t)=\hat{A} \exp [i k(x-c t)], \quad \varphi(x, t)=\hat{B} \exp [i k(x-c t)],
$$

substitution of (19) into (17) and (18) yields a set of characteristic equations,

$\left(-\rho c^{2}+\beta G+E \varepsilon_{0}+\frac{2 \gamma}{R_{0}}\right) k^{2} \hat{A}+i \beta G k \hat{B}=0$,

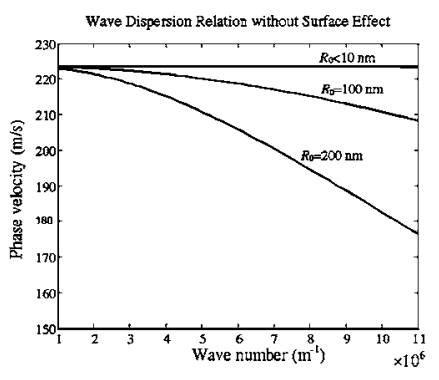

(a)

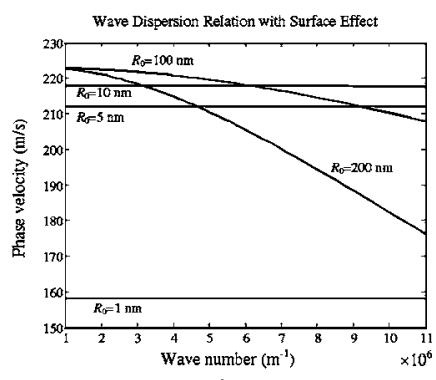

(b)
FIG. 3. Variation of the phase velocity vs the wave number at varying nanofiber radius (longitudinal wave propagation).

$-i A\left(\beta G+E \varepsilon_{0}+\frac{2 \gamma}{R_{0}}\right) k \hat{A}+\left(-\rho I k^{2} c^{2}+\beta G A+E I k^{2}\right) \hat{B}=0$.

Nontrivial solution of the set of linear algebraic equations above leads to the wave dispersion relation as

$$
\begin{gathered}
\rho^{2} I k^{2} c^{4}-\rho\left[\left(\beta G+E \varepsilon_{0}+\frac{2 \gamma}{R_{0}}\right) I k^{2}+\left(\beta G A+E I k^{2}\right)\right] c^{2} \\
+\left(\beta G+E \varepsilon_{0}+\frac{2 \gamma}{R_{0}}\right) E I k^{2}=0 .
\end{gathered}
$$

The phase velocity $c$ can be extracted by solving the quadratic equation above with respect to $c^{2}$,

$$
c=\sqrt{\frac{-a_{12} \pm \sqrt{a_{12}^{2}-4 a_{11} a_{13}}}{2 a_{11}}},
$$

where $\quad a_{11}=\rho I k^{2}, \quad a_{12}=-\rho\left[\left(\beta G+E \varepsilon_{0}+2 \gamma / R_{0}\right) I k^{2}+(\beta G A\right.$ $\left.\left.+E I k^{2}\right)\right]$, and $a_{13}=\left(\beta G+E \varepsilon_{0}+2 \gamma / R_{0}\right) E I k^{2}$. It can be found that Eq. (23) usually yields two phase velocities for each wave number, of which the lower one corresponds to the flexural wave, and the upper one accords to the transverse shear wave. In the limiting case of $\varepsilon_{0} \rightarrow 0$ and $\gamma \rightarrow 0$, the results above recover those given by the traditional Timoshenko beam theory. ${ }^{29}$

\section{Numerical examples}

In Secs. II A and II B wave equations have been derived for determining the dispersion relations of the longitudinal and flexural waves propagating in straight nanofibers/ nanowires. In this section, based on the wave dispersion relations (5) and (23), numerical calculations are conducted to examine the variation of the phase velocity versus the wave number at varying nanofiber/nanowire diameter. Specifically, the material used is assumed as typical isotropic elastomer with the physical properties: Young's modulus $E \sim 100 \mathrm{MPa}, \quad$ Poisson's ratio $\nu \sim 0.5$, mass density $\rho \sim 2000 \mathrm{~kg} / \mathrm{m}^{3}$, and surface tension (surface energy) $\gamma \sim 0.05 \mathrm{~N} / \mathrm{m}$. For an isotropic material, the shear modulus is $G=E /[2(1+\nu)] \sim 33.33 \mathrm{MPa}$, and the longitudinal wave speed in bulk material is $c=\sqrt{E / \rho} \sim 223.6 \mathrm{~m} / \mathrm{s}$. In the calculation, the prestrain is simply assumed as zero, i.e., $\varepsilon_{0}=0$.

Figure 3 shows the longitudinal wave dispersion relations for nanofibers of radii of $1,5,10,100$, and $200 \mathrm{~nm}$. It can be found that surface tension remarkably influences the 
Wave Dispersiont Relation without Surface Effect

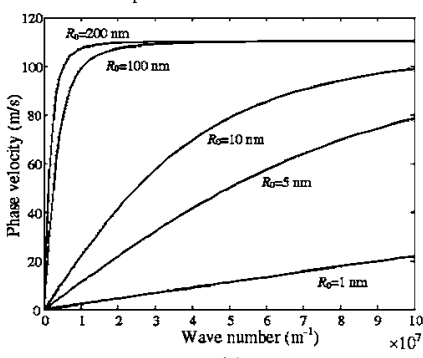

(a)

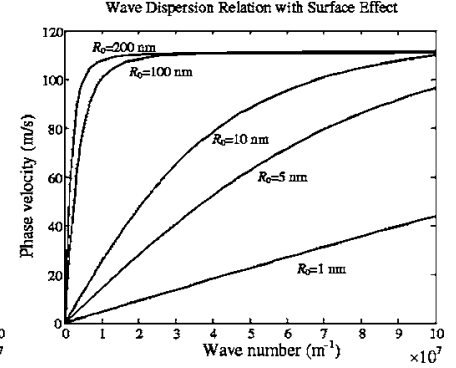

FIG. 4. Variation of the phase velocity vs the wave number at varying nanofiber radius (flexural wave propagation).

dispersion relation at small fiber diameters. In the range of small nanofiber diameters, e.g., $<20 \mathrm{~nm}$, the phase velocity decreases rapidly with the decrease of the nanofiber diameter, and in this range the phase velocity is almost independent of the wave number, i.e., nondispersive. By comparison with those calculated without the surface effect [see Fig. $3(\mathrm{a})$ ], it can be observed that at great fiber diameters, surface tension does not have appreciable effect, i.e., the present results recover those predicted by the classic Rayleigh-Love rod equation.

By using the wave dispersion relation (23), Fig. 4 shows the numerical results for the elastomer nanofibers with varying radius aforementioned. It can be found that the wave dispersion curves for nanofibers with and without surface effect have the similar growth trend. However, at very small fiber diameters, nanofibers with surface effect tend to have much greater phase velocities at fixed wave numbers. For example, in the case of nanofibers with a radius of $10 \mathrm{~nm}$ at the wave number of $10^{8} \mathrm{~m}^{-1}$, the corresponding phase velocity with surface effect is about $10 \%$ higher than that without surface effect. However, when the nanofiber radius decreases to $1 \mathrm{~nm}$, the phase velocity with surface effect is about $100 \%$ higher than that without surface effect at the wave number of $10^{8} \mathrm{~m}^{-1}$. Therefore, when dealing with the wave propagation in nanofibers and nanowires at very small diameters, surface effect should be taken into account in order to accurately describe these unique dynamic phenomena.

\section{SUMMARY}

In this study we have developed the elastic wave equations governing the longitudinal and flexural waves propagating in nanofibers and nanowires within the framework of continuum mechanics. Modified Euler-Bernoulli and Rayleigh-Love rod equations and modified Timoshenko beam equation have been obtained to take into account the effect of the surface tension/stress in the first order. Wave dispersion relations have been obtained in closed form. $\mathrm{Nu}-$ merical calculations have been performed to show that surface tension/stress has appreciable influence on the wave propagation in nanofibers and nanowires at small diameters. For longitudinal waves, surface tension/stress results in appreciable decrease of the phase velocity at fixed wave number, while for flexural waves, the surface tension/stress leads to observable increase of the phase velocity at given wave number. The present study first indicated the surface effect on the wave propagation in nanofibers and nanowires that can be employed as the theoretical basis of dynamic characterization of nanofibers/nanowires and nanodevice design and analysis.

\section{ACKNOWLEDGMENTS}

Partial support of this work by NSF, AFOSR, and ARO/ ARL is gratefully acknowledged.

${ }^{1}$ K. Sieradzki and R. C. Cammarata, Phys. Rev. Lett. 62, 2005 (1989).

${ }^{2}$ P. Gumbsch and M. S. Daw, Phys. Rev. B 44, 3934 (1991).

${ }^{3}$ F. H. Streitz, R. C. Cammarata, and K. Sieradzki, Phys. Rev. B 49, 10699 (1994).

${ }^{4}$ R. C. Cammarata, Prog. Surf. Sci. 46, 1 (1994).

${ }^{5}$ R. E. Miller and V. B. Shenoy, Nanotechnology 11, 139 (2000).

${ }^{6}$ S. Cuenot, C. Fretigny, S. Demoustier-Champagne, and B. Nysten, Phys. Rev. B 69, 165410 (2004)

${ }^{7}$ H. Y. Liang, M. Upmanyu, and H. C. Huang, Phys. Rev. B 71, 241403 (2005).

${ }^{8}$ A. Sharma and G. Reiter, J. Colloid Interface Sci. 178, 383 (1996).

${ }^{9}$ X. F. Wu and Y. A. Dzenis, J. Phys. D 38, 2848 (2005).

${ }^{10}$ J. Yoon, C. Q. Ru, and A. Mioduchowski, J. Appl. Phys. 93, 4801 (2003).

${ }^{11}$ L. F. Wang, H. Y. Hu, and W. L. Guo, Nanotechnology 17, 1408 (2006).

${ }^{12}$ Q. Wang, Int. J. Struct. Stab. Dyn. 6, 285 (2006).

${ }^{13}$ Q. Wang and V. K. Varadan, Int. J. Solids Struct. 43, 254 (2006).

${ }^{14}$ L. F. Wang and H. Y. Hu, Phys. Rev. B 71, 195412 (2005).

${ }^{15} \mathrm{X}$. Wang and H. Cai, Acta Mater. 54, 2067 (2006).

${ }^{16}$ K. Dong and X. Wang, Nanotechnology 17, 2773 (2006).

${ }^{17}$ C. Y. Li and T. W. Chou, Phys. Rev. B 73, 245407 (2006).

${ }^{18}$ C. J. Strobl, C. Schaflein, U. Beierlein, J. Ebbecke, and A. Wixforth, Appl. Phys. Lett. 85, 1427 (2004).

${ }^{19}$ D. H. Reneker and I. Chun, Nanotechnology 7, 216 (1996).

${ }^{20}$ Y. Dzenis, Science 304, 1917 (2004).

${ }^{21}$ S. Y. Chew, Y. Wen, Y. Dzenis, and K. W. Leong, Current Pharmaceutical Design 12, 4751 (2006).

${ }^{22}$ D. Li and Y. N. Xia, Adv. Mater. (Weinheim, Ger.) 16, 1151 (2004).

${ }^{23}$ C. B. Huang, S. L. Chen, C. L. Lai, D. H. Reneker, H. Qiu, Y. Ye, and H. Q. Hou, Nanotechnology 17, 1558 (2006).

${ }^{24}$ E. P. S. Tan and C. T. Lim, Rev. Sci. Instrum. 75, 2581 (2004).

${ }^{25}$ E. Zussman, M. Burman, A. L. Yarin, R. Khalfin, and Y. Cohen, J. Polym. Sci., Part B: Polym. Phys. 44, 1482 (2006).

${ }^{26}$ E. P. S. Tan and C. T. Lim, Compos. Sci. Technol. 66, 1102 (2006).

${ }^{27}$ J. E. Muller, M. Wuttig, and H. Ibach, Phys. Rev. Lett. 56, 1583 (1986).

${ }^{28}$ T. Kaneko, J. Phys. D 8, 1927 (1975).

${ }^{29}$ J. D. Achenbach, Wave Propagation in Elastic Solids (Elsevier, Amsterdam, 1999). 